World Nutrition 2018;9(3):284-291

\title{
Negative impact of fructose overconsumption on health
}

\author{
Author \\ Heba Rady Salem (MD) \\ Institution \\ Physiology Department, Faculty of Medicine, Menoufia University, Shebin El-Kom, Menoufia, \\ Egypt \\ Funds: None \\ Conflict of Interests: None \\ e-mail: heba.salem.12@med.menofia.edu.eg
}




\section{Introduction}

Light has been shed recently on fructose, because its consumption has increased significantly in diets in recent years in parallel with the increase in intake of sucrose and high fructose corn syrup (HFCS).

Fructose, or fruit sugar, is a natural monosaccharide that is found in fruits, honey and some vegetables. However, currently its main source in our diet comes from sucrose (table sugar; a disaccharide of fructose and glucose), and HFCS (a type of artificial sugar made from corn starch, that contains either $42 \%$ or $55 \%$ fructose combined with glucose) (White, 2008).

HFCS was introduced in 1970 as a sweetener in pastries, yogurt, ice cream, canned fruits, jams and soft drinks. Fructose is preferred in food industry because it is at least 1.5 times sweeter than sucrose, much cheaper, and has better functional characteristics (Dornas et al., 2015).

Fructose overconsumption has been widely considered to be a contributor to the worldwide epidemics of obesity and diabetes and their associated cardiometabolic risks (Hannou et al., 2018). This article will illuminate the negative impact of fructose overconsumption on different body systems based on clinical and experimental studies.

\section{Impact on metabolism}

Fructose, glucose and sucrose are three types of sugar that contain the same number of calories per gram. But fructose may be the worst for health. The body converts fructose to glucose in the liver to use for energy. Excess fructose places a burden on the liver, which may lead to a series of metabolic disorders (Laughlin, 2014).

Initially, fructose attracted great interest as a sweetener for diabetic patients due to its lower glycemic index than glucose (Uusitupa, 1994). However, excessive fructose contributes negatively to blood glucose homeostasis by causing insulin resistance, a condition that can result in type 2 diabetes (Basciano et al., 2005).

Also, there is rising evidence in both animal and human studies that high dietary fructose intake is a significant risk factor for the development of metabolic syndrome and its related complications (Tappy et al., 2010). The current hypotheses regarding the mechanisms by which fructose promotes metabolic syndrome focus on its lipogenic nature. Excessive intake of fructose has been shown to increase de novo lipogenesis. The induction of lipogenesis would increase deposition of triglycerides in adipose tissue and ectopic tissues, such as liver and muscle, eventually resulting in impaired insulin signaling and dyslipidemia (Ter Horst and Serlie, 2017). This is a huge problem especially among chronic soda drinkers. The lipogenic nature of fructose compared to glucose is illustrated in Figure 1 (Health Jade Team, 2018).

Sucrose and HFCS are widely indicated to be key factors in today's obesity epidemic (Lustig et al., 2012). Also, high fructose intake is a major mediator of nonalcoholic fatty liver disease in experimental (Abdel-Razek et al., 2017) and clinical studies (Jensen et al., 2018).

In addition, studies have shown that fructose can induce hyperuricemia; this supports work that has demonstrated an association between a high consumption of soft drinks and an increase in serum uric acid concentrations (Wang et al., 2012). 


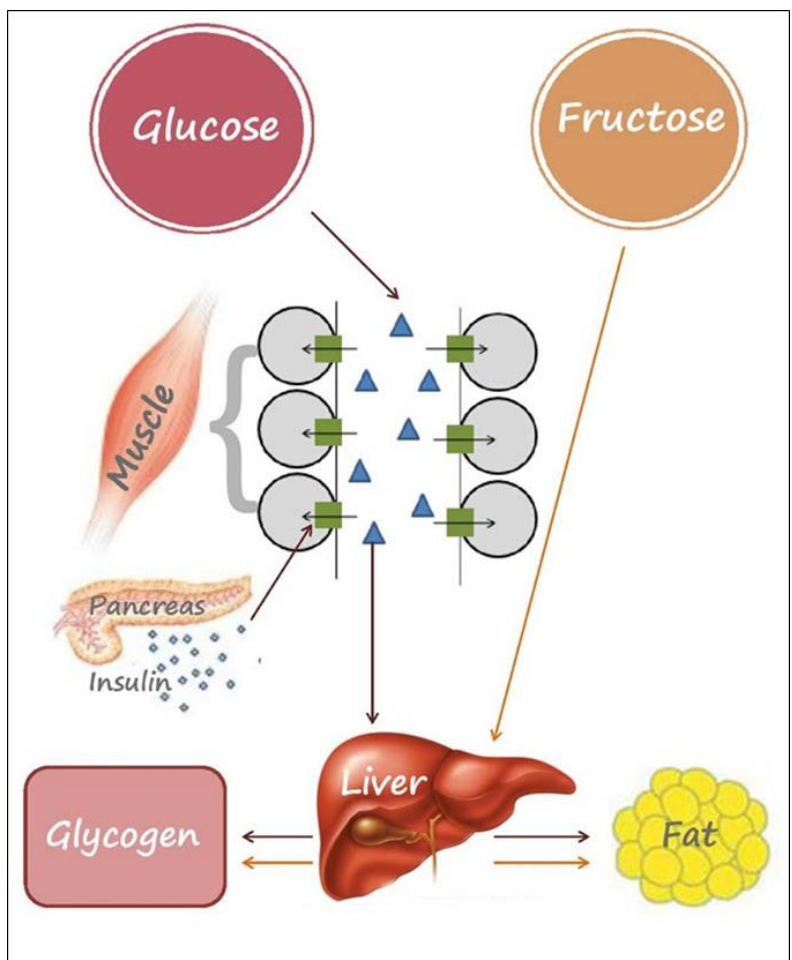

Figure (1): Fructose versus glucose metabolism (Health Jade Team, 2018)

There are numerous mechanisms to explain why fructose might be more challenging compared to the consumption of the same amount of glucose, some of which are summarized in Figure 2. Fructose consumption has been suggested to induce hepatic and systemic inflammation via increased intestinal permeability and the translocation of endotoxin from the gut and into circulation. Also, an increase in de novo lipogenesis can increase production of fetuin-A, a protein secreted by the liver and adipose tissue, and in turn lead to increased activity of inflammatory pathways in adipose tissue (Patel, 2018). In addition to inflammation, fructose overconsumption may also increase injurious substances called advanced glycation end products, which may cause cell injury and promote aging (Dhar et al., 2008).

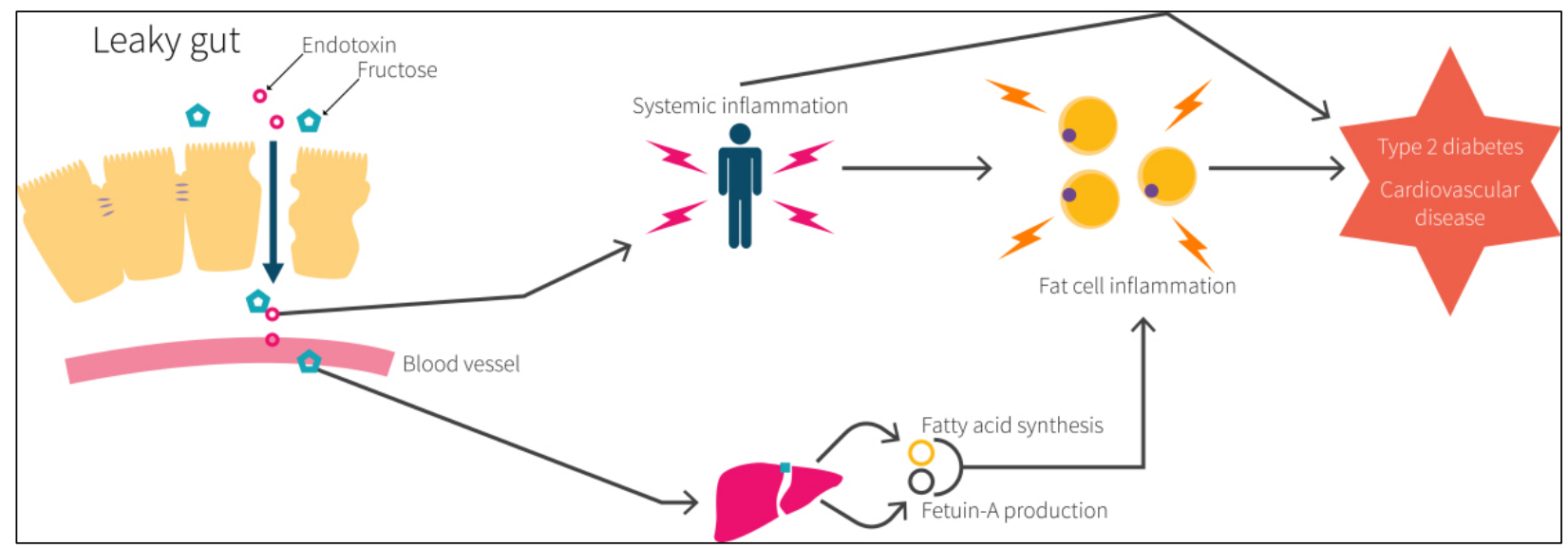

Figure (2): The mechanisms by which fructose can induce inflammation (Patel, 2018) 


\section{Impact on the cardiovascular system}

Intake of HFCS sweetened soft drinks and juices has been reported to be associated with coronary heart disease in US adults. This could be attributed to the underlying fructose malabsorption, which may contribute to the intestinal in situ formation of pro-inflammatory advanced glycation endproducts, which are eventually absorbed and induce inflammation of the coronary arteries (DeChristopher et al., 2017). High fructose consumption also reduces the size of low-density lipoprotein particles, a risk factor in heart disease (Bray, 2007).

Also, high fructose consumption can induce hypertension. However, the exact underlying mechanism is uncertain. Several possibilities have been proposed including increased sympathetic nervous system activity, elevated circulating catecholamines, enhanced renin-angiotensin system activity and angiotensin-II levels, increased sodium reabsorption, impaired endotheliumdependent relaxation, and increased secretion of endothelin-1. All these factors may contribute to an increased vascular tone and endothelial dysfunction. Moreover, several animal studies have shown that high-fructose diets up-regulate sodium and chloride transporters stimulating sodium and chloride absorption, resulting in a state of salt overload that increases blood pressure (Dornas et al., 2015).

\section{Impact on the kidney}

High fructose intake may also be a risk factor for kidney disease that includes glomerular hypertension, renal inflammation, and tubulointerstitial injury in experimental studies (Johnsonet al., 2010). There is a strong association between drinking too much soda and developing kidney disease. Rats fed extremely high-sugar diets developed enlarged kidneys and poor kidney function (Karalius and Shoham, 2013).

\section{Impact on the gastrointestinal system}

High levels of fructose in the diet alter the gut microbiota and increase intestinal permeability (Payne et., 2012). This may be involved in non-alcoholic fatty liver disease (Lambertz et al., 2017) as well as preceding the development of metabolic endotoxemia, inflammation, and lipid accumulation, ultimately leading to hepatic steatosis and normal-weight obesity (Do et al., 2018).

\section{Impact on the nervous system}

Increased sugar consumption is associated with cognitive impairment in experimental studies, especially worsened hippocampal memory function. Rats on a high sugar/low fat, or a high sugar/high fat diet show hippocampal-dependent memory deficits. This relation appears to be mediated by increased hippocampal inflammation (Freeman et al., 2018).

Studies in humans support these findings. High intake of added sugar, but not natural fructose from fruits, was associated with decreased cognitive performance in middle-aged and older Puerto Rican adults (Stoianov and Adeli, 2014). Also, greater relative carbohydrate intake predicted a heightened risk of mild cognitive impairment or dementia in elderly people, while carbohydrate intake in school children was negatively associated with nonverbal intelligence tests (Lane and Cha, 2009). 
Compared with glucose, fructose may be a weaker suppressor of appetite. The ingestion of fructose results in smaller increases in plasma insulin levels and greater brain responses to food signals in the visual cortex and left orbital frontal cortex. Fructose consumption also leads to greater hunger and desire for food. These findings suggest that ingestion of fructose relative to glucose activates brain regions involved in attention and reward processing and may promote feeding behavior (Luo et al., 2015).

Intermittent access to sugar can lead to changes that resemble the effects of a substance of abuse, according to one study, in which rats with 12 hours of access to sugar followed by 12 hours of food deprivation showed bingeing, withdrawal, craving, and cross sensitization to drugs of abuse (Hoebel et al., 2009).

Also, HFCS can impair dopamine function in the absence of weight gain or increased fat consumption. Reduced dopamine function has been implicated in compulsive behaviors and reduced energy expenditure and insulin dysregulation (Meyers et al., 2017).

\section{Impact on fertility}

In rats there is evidence that fructose overconsumption has a negative impact on female fertility. Broughton and Moley (2017) reported that obesity linked to high fructose consumption is associated with multiple adverse reproductive outcomes in females.

Ko et al. (2017) reported that relatively short-term HFCS treatment in rats could induce a status of reproductive senescence by inducing changes in length of estrous cycle and altered ovarian and uterine histology. As the estrous cycle is lengthened, the rats became acyclic.

\section{Impact of fructose consumption in pregnancy and lactation}

In rodents, perinatal exposure (before birth and during lactation) to excess maternal consumption of added sugars, including fructose and sucrose, has detrimental effects on the offspring, mainly on adiposity, plasma FFAs, hepatic fat content and hepatic lipid composition. While, these results cannot be directly translated to humans, they strongly suggest that controlling maternal consumption of added fructose may have a positive impact on the short- and long-term metabolic health of the offspring (Toop et al., 2017).

Maternal nutrition during the lactation period seems to be as important as nutrition during pregnancy, as suggested by Berger et al. (2018), who revealed that mothers' consumption of HFCS-sweetened beverage significantly increased breast milk fructose concentration, which persisted for five hours, with no effect on breast milk glucose or lactose. But, the consequences of the habitual elevation of breast milk fructose needs further research.

The indirect effects of fructose overconsumption on the next generations are still unknown. Can the metabolic change produced by maternal intake of excess fructose will be reversible or will it persist into adulthood? Considering the huge increase in sugar consumption in the world, this is a very important question.

Furthermore, higher sugar-sweetened beverage and fructose intake during pregnancy and in early childhood is associated with childhood asthma (Wright et al., 2018). 


\section{Conclusions}

The Dietary Guidelines for Americans 2015-2020 recommend that added sugars from any source, including HFCS, should be limited to no more than 10 percent of calories a day. For someone consuming 2,000 calories a day, that's 50 grams of added sugar. WHO has indicated that additional health benefits would follow from consuming less that $5 \%$ of calories ( 25 grams) as sugars (Joviak, 2018).

Fruit is good for you, while HFCS is not. As the sugars found in whole fruit have been shown to be harmless, since it's accompanied by healthful micronutrients and antioxidants, as well as fiber, which slows absorption of fructose. Plus, you would have to eat several servings of fruit to get as much fructose as in a can of soda. But limit fruit juices to no more than a cup a day; some contain nearly as much fructose as soda. There is evidence that many substances found in natural fruits, such as flavanols, epicatechin, vitamin $\mathrm{C}$ and other antioxidants may also protect against fructoseinduced metabolic syndrome (Vasdev et al., 2002).

Fructose consumption has increased significantly in diets over the last years. However, high dietary fructose intake has proved to have a negative impact on health, including metabolic syndrome and its associated complications, non-alcoholic fatty liver disease, hypertension, kidney disease and cognitive impairment. It may even affect fertility, pregnancy, and perinatal exposure may affect offspring. Fructose intake should be limited based on the major health implications of high levels of consumption. 


\section{References}

Abdel-Razek HA, Hanna GS, Abou-Elnour ES, et al. 2017. Effect of exercise and melatonin on fructose-induced hepatic dysfunction in a metabolic syndrome rat model. Menoufia Med J 30:286-96. DOI: 10.4103/1110-2098.211506.

Basciano H, Federico L and Adeli K 2005. Fructose, insulin resistance, and metabolic dyslipidemia. Nutr Metab (Lond). 21;2(1):5. DOI:10.1186/1743-7075-2-5.

Berger PK, Fields DA, Demerath EW, et al. 2018. High-fructose corn-syrup-sweetened beverage intake increases 5-hour breast milk fructose concentrations in lactating women. Nutrients 10, 669; DOI:10.3390/nu10060669.

Bray GA. 2007. How bad is fructose? The American Journal of Clinical Nutrition 86:(4): 895896, https://doi.org/10.1093/ajcn/86.4.895. Broughton DE and Moley KH 2017. Obesity and female infertility: potential mediators of obesity's impact. Fertil Steril 107(4):840-847. DOI: 10.12717/DR.2017.21.2.151.

DeChristopher LR, Uribarri and Tucker KL 2017. Intake of high fructose corn syrup sweetened soft drinks, fruit drinks and apple juice is associated with prevalent coronary heart disease, in U.S. adults, ages 45-59 y. BMC Nutrition 3(1):51. DOI 10.1186/s40795-017-0168-9.

Dhar A, Desai K, Kazachmov M, et al. 2008. Methylglyoxal production in vascular smooth muscle cells from different metabolic precursors. Metabolism 57(9):1211-20. DOI: 10.1016/j.metabol.2008.04.014.

Dietary Guidelines for Americans 2015-2020. Website. Accessed September 10, 2018. https://health.gov/dietaryguidelines/2015/guidelines/

Do $\mathrm{MH}$, Lee E, Oh M, et al. 2018. High-Glucose or -Fructose Diet Cause Changes of the Gut Microbiota and Metabolic Disorders in Mice without Body Weight Change. Nutrients 10(6):761. DOI:10.3390/nu10060761.

Dornas WC, de Lima WG, Pedrosa ML, et al. 2015. Health Implications of High-Fructose Intake and Current Research. Advances in Nutrition 6(6): 729-737. DOI:10.3945/an.114.008144.

Freeman CR, Zehra A, Ramirez V et al. 2018. Impact of sugar on the body, brain, and behavior. Front Biosci (Landmark Edition).1;23:2255-2266.

Hannou SA, Haslam DE, Mckeown NM, et al. 2018. Fructose metabolism and metabolic disease. J Clin Invest 1;128(2):545-555. DOI: 10.1172/JCI96702.

Health Jade Team. How Your Body Metabolizes Sugar. Website accessed October,29,2018. https:/healthjade.com/how-your-body-metabolizes-sugar/

Hoebel BG, Avena NM, Bocarsly ME, et al. 2009. Behavioral and Circuit Model Based on Sugar Addiction in Rats. Journal of Addiction Medicine. 3(1):33-41. DOI:10.1097/ADM.0b013e31819aa621.

Jensen T, Abdelmalek MF, Sullivan S, et al. 2018. Fructose and Sugar: A Major Mediator of Nonalcoholic Fatty Liver Disease. Journal of Hepatology. 68(5):1063-1075. DOI:10.1016/j.jhep.2018.01.019.

Johnson RJ, Sanchez-Lozada LG and Nakagawa T 2010. The Effect of Fructose on Renal Biology and Disease. JASN 21 (12) 2036-2039; DOI: https://doi.org/10.1681/ASN.2010050506.

Joviak M. 2018. Examination of differences in added sugar consumption and urinary sugar excretion between post-menopausal women with healthy weight and post-menopausal women with obesity (Doctoral dissertation, The Ohio State University).Karalius VP and Shoham DA 2013. Dietary Sugar and Artificial Sweetener Intake and Chronic Kidney Disease: A Review. Advances in Chronic Kidney Disease 20 (2):157-164. DOI: https://doi.org/10.1053/j.ackd.2012.12.005 
Ko E, Kim H, Kim Y, et al. 2017. Effect of high fructose corn syrup (hfcs) intake on the female reproductive organs and lipid accumulation in adult rats. Dev. Reprod 21(2):151-156. https://doi.org/10.12717/DR.2017.21.2.151.

Lambertz J, Weiskirchen S, Landert S, et al. 2017. Fructose: A dietary sugar in crosstalk with microbiota contributing to the development and progression of non-alcoholic liver disease. Frontiers in immunology. 19;8:1159. https://doi.org/10.3389/fimmu.2017.01159.

Lane MD and Cha SH 2009. Effect of glucose and fructose on food intake via malonyl-CoA signaling in the brain. Biochem Biophys Res Commun 382(1): 1-5. DOI: 10.1016/j.bbrc.2009.02.145.

Laughlin MR 2014. Normal roles for dietary fructose in carbohydrate metabolism. Nutrients. 5;6(8):3117-29. DOI: 10.3390/nu6083117.

Luo S, Monterosso JR, Sarpelleh K, et al. 2015. Differential effects of fructose versus glucose on brain and appetitive responses to food cues and decisions for food rewards. Proc Natl Acad Sci U S A. 112(20):6509-14. DOI: 10.1073/pnas.1503358112.

Lustig RH, Schmidt LA and Brindis CD 2012. Public health: The toxic truth about sugar. Nature. 1;482(7383):27-9. DOI: 10.1038/482027a.

Meyers AM, Mourra D and Beeler JA 2017. High fructose corn syrup induces metabolic dysregulation and altered dopamine signaling in the absence of obesity. PLoS ONE 12(12): e0190206. https://doi.org/10.1371/journal.pone.0190206

Patel K. 2016. Fructose vs Glucose vs HFCS, Examine.com, published on 19 September 2016, last updated on 22 October 2018, https://examine.com/nutrition/fructose-vs-glucose-vs-hfcs/

Payne AN, Chassard C and Lacroix C. 2012. Gut microbial adaptation to dietary consumption of fructose, artificial sweeteners and sugar alcohols: implications for host-microbe interactions contributing to obesity. Obesity reviews 13(9):799-809. DOI: 10.1111/j.1467789X.2012.01009.x.

Stoianov A and Adeli K. 2014. Central and metabolic effects of high fructose consumption: evidence from animal and human studies, Nutr Food Sci Research 1(2), 3-9. http://nfsr.sbmu.ac.ir/article-1-64-en.html

Tappy L, Lê KA, Tran C, et al. 2010. Fructose and metabolic diseases: New findings, new questions. Nutrition 26:1044-1049. DOI:10.1016/j.nut.2010.02.014.

Ter Horst KW and Serlie MJ. 2017. Fructose Consumption, Lipogenesis, and Non-Alcoholic Fatty Liver Disease. Nutrients, 9(9):981. DOI:10.3390/nu9090981.

Toop CR, Muhlhausler BS, O'Dea K, et al. 2017. Impact of perinatal exposure to sucrose or high fructose corn syrup (HFCS-55) on adiposity and hepatic lipid composition in rat offspring. J Physiol 595: 4379-4398. DOI: 10.1113/JP274066.

Uusitupa MIJ. 1994. Fructose in the diabetic diet. Am J Clin Nutr 59(3 Suppl):753S-7S. DOI: 10.1093/ajcn/59.3.753S.

Vasdev S, Gill V, Parai S, et al. 2002. Dietary vitamin E and C supplementation prevents fructose induced hypertension in rats. Mol Cell Biochem 241:107-114.

Wang DD, Sievenpiper JL, de Souza RJ, et al. 2012. The effects of fructose intake on serum uric acid vary among controlled dietary trials. J Nutr 142:916-23. DOI: 10.3945/jn.111.151951.

White JS. 2008. Straight talk about high-fructose corn syrup: what it is and what it ain't. Am J Clin Nutr 88(6):1716S-1721S. DOI: 10.3945/ajcn.2008.25825B.

Wright LS, Rifas-Shiman SL, Oken E, et al. 2018. Prenatal and early life fructose, fructosecontaining beverages, and midchildhood asthma. Ann Am Thorac Soc 15(2):217-224. doi: 10.1513/AnnalsATS.201707-5300C. 